\title{
In-vitro assessment of coronary artery stents in 256-multislice computed tomography angiography
}

Florian André ${ }^{*}$, Dirk Müller ${ }^{2}$, Grigorios Korosoglou', Waldemar Hosch ${ }^{3}$, Hans-Ulrich Kauczor ${ }^{3}$, Hugo A Katus ${ }^{1}$ and Henning Steen ${ }^{1}$

\begin{abstract}
Background: The important detection of in-stent restenosis in cardiovascular computed tomography (CT) is still challenging. The first study assessing the in-vitro stent lumen visualization of the state of the art 256-multislice CT (256-MSCT), which was performed by our research group, yielded promising results. As the applied technical approach is not suitable for daily routine, we assessed the capability of the 256-MSCT and its different reconstruction kernels for the coronary stent lumen visualization employing a clinically applicable technique in a phantom study.

Results: The XCD kernel showed significantly lower artificial lumen narrowing (ALN) values (overall ALN $<40 \%$ ) than the other reconstruction kernels (CC, CD, XCB) irrespective of the stent caliber. The ALN of coronary stents with a diameter $>3 \mathrm{~mm}$ was significantly lower than of stents with a smaller caliber. The ALN difference between stents with a diameter of $3 \mathrm{~mm}$ and smaller ones was not statistically significant. Yet, the lumen visualization of the smaller stents was impaired by a halo effect. The XCD kernel showed more constant attenuation values throughout the different stent diameters than the other reconstruction kernels.

Conclusions: The 256-MSCT provides a good lumen visualization of coronary stents with a diameter $>3 \mathrm{~mm}$. The assessment of stents with a diameter of $3 \mathrm{~mm}$ seems feasible but has to be validated in further studies. The clinical evaluation of smaller stents cannot be recommended so far. The XCD kernel showed the best lumen visualization and should therefore be applied in addition to the standard cardiac reconstruction kernels when assessing coronary artery stents using 256-MSCT.
\end{abstract}

Keywords: CT angiography, Coronary artery stents, In-stent restenosis, Heart, Technology assessment

\section{Background}

In a previous study we could show that the 256-multislice computed tomography (MSCT) yields promising results for the visualization of coronary artery stents using a technical approach [1]. Therefore, we provide an update on this trial employing a clinically applicable technique.

Currently, percutaneous coronary intervention (PCI) and stent placement are the leading coronary revascularization therapies in patients with coronary artery disease (CAD). Approximately 492000 patients underwent PCI

\footnotetext{
* Correspondence: florian.andre@med.uni-heidelberg.de

'Department of Cardiology, University of Heidelberg, Im Neuenheimer Feld 410, Heidelberg 69120, Germany

Full list of author information is available at the end of the article
}

procedures in the US in 2010 [2]. The clinical use of drug eluting stents (DES) instead of bare metal stents (BMS) reduces the rates of in-stent restenosis (ISR) from about $20-30 \%$ to less than $10 \%[3,4]$. But despite the increasing amount of DES coronary interventions, ISR and stent thrombosis are still a major limitation of $\mathrm{PCI}$, leading to an increased morbidity and mortality in patients with coronary stents [5]. Moreover, a considerable number of these patients have to undergo conventional coronary angiography (CA) to exclude ISR. In addition to bearing the risks of an invasive procedure, CA is linked to considerable health care costs. Hence, a non-invasive alternative may be of great clinical interest as well as of socio-economic benefit.

\section{() Biomed Central}


Nowadays, the angiography of the coronary arteries using MSCT is a non-invasive imaging modality for the reliable diagnosis and assessment of CAD yielding a sensitivity of $97 \%-99 \%$ and a specificity of $88 \%-89 \%$ $[6,7]$. Yet, the evaluation of coronary artery stents is challenging due to artifacts like beam hardening and partial volume effects caused by the metal of the stent struts and sporadically by the stents' radio-opaque markers. First attempts to visualize the lumen of coronary artery stents using CT were made about 20 years ago using electron beam CT without reaching an image quality that was sufficient for analysis [8]. Since then, the MSCT technology has developed tremendously and new scanner generations with 4,16 and 40 slices have been assessed for ISR detection $[9,10]$. With the introduction of the 64-MSCT the reliable visualization of ISR seemed to be achievable [11]. Yet, several reviews came to the conclusion that the diagnostic performance of the 64-MSCT was not sufficient in patients with implanted coronary artery stents [12-14].

The introduction of the latest CT scanner generations including dual source CT (DSCT) with high temporal resolution on the one hand and single-source MSCT with wide z-axis coverage of 256 or 320 slices on the other hand arose the hope for reliable stent lumen assessment. In addition, previous studies showed that stent-dedicated, sharper reconstruction kernels were able to reduce stent artifacts and improve stent lumen visualization $[1,15,16]$.

In the first study using the 256-MSCT for the assessment of coronary artery stents, our research group got promising results [1]. We applied a full width at half maximum algorithm to measure the extent of the impaired in-stent lumen visualization objectively. Yet, this technical approach is associated with a high effort and is, therefore, impractical for a daily-base clinical use. Furthermore, the obtained results might reflect technical values rather than visual assessment as performed in clinical routine - hence the need for an assessment of the stent lumen visualization capability of the 256-MSCT using a clinically applicable approach. In this study, we sought to investigate coronary stent visualization and in-stent lumen assessment using various kernels in a state of the art 256-MSCT by employing a visual analysis technique.

\section{Methods}

We included 51 stents of different diameters, materials, strut designs and manufacturers in this study. A summary is provided in Table 1.

The stents were assorted into three groups according to their diameters: group A: stents $<3 \mathrm{~mm}$, group $\mathrm{B}$ : stents $=3 \mathrm{~mm}$, group $C$ : stents $>3 \mathrm{~mm}$. The stents were expanded at the nominal pressure of their respective delivery system into a vessel phantom, which was made of plastic tubes with a wall thickness of $0.5 \mathrm{~mm}$. Stents of group A and B were placed in tubes with an inner diameter of $3 \mathrm{~mm}$, stents of group $C$ in tubes with an inner diameter of $4 \mathrm{~mm}$. The tubes were filled with contrast agent and saline (Ultravist 370, Bayer HealthCare, Leverkusen, Germany) providing a radio-density of approximately $250 \mathrm{HU}$. Afterwards, the tubes were closed at both ends and placed into a plastic basin. This was filled with an oil-iodine solution attaining a radiodensity of approximately $-70 \mathrm{HU}$ according to the epicardial fat tissue. The basin was placed into the gantry, thus aligning the tubes in a parallel manner to the scanner's z-axis.

Imaging was performed on a 256-slice CT scanner (Brilliance iCT, Philips Healthcare, Cleveland, OH, USA) applying the following parameters: helical mode, collimation $=2 \times 128 \times 0.625 \mathrm{~mm}$, tube voltage $=120 \mathrm{kV}$, tube current-time product $=800 \mathrm{mAs}_{\text {eff, }}$ pitch $=0.18$, tube rotation time $=270 \mathrm{~ms}$, field of view $=180 \mathrm{~mm}$.

All images were reconstructed at $75 \%$ of an artificial ECG-signal with a heart rate of $60 \mathrm{bpm}$ using a matrix of $512 \times 512$. Four different convolution kernels were applied: a) Xres detailed stent (XCD), b) cardiac sharp $(C C), c)$ cardiac detailed stent (CD) and Xres standard (XCB). While $\mathrm{XCB}$ is the standard kernel for the reconstruction of coronary arteries, $\mathrm{XCD}$ and $\mathrm{CD}$ are dedicated kernels for the visualization of stents.

The analysis was carried out on a dedicated CT postprocessing workstation (Extended Brilliance Workspace V 3.5.3.1020, Philips Healthcare, Cleveland, OH, USA) with a window center of $300 \mathrm{HU}$ and a width of $1200 \mathrm{HU}$.

\section{Artificial lumen narrowing}

Artificial lumen narrowing (ALN) was defined as the difference between the nominal stent diameter and the one measured visually using the electronic diameter provided by the workstation's software. Measurements were performed in axial orientation in a proximal, middle and distal part of the stent and a mean value was calculated.

\section{Stent and tube lumen attenuation}

As the measured radio-density inside the stent lumen can be influenced by artifacts like beam hardening or partial volume effects, we measured two values: stent lumen attenuation (SLA) and tube lumen attenuation (TLA). The quantification was carried out in a longitudinal view by a region-of-interest (ROI) technique. SLA was assessed by placing the largest possible ROI inside the lumen of the deployed stent omitting strut artifacts. For TLA two ROIs were positioned inside the tube lumen outside both sides of the stent and a weighted mean value was calculated. 
Table 1 List of coronary artery stents

\begin{tabular}{|c|c|c|c|c|c|c|c|}
\hline Manufacturer & Name & $\emptyset(\mathrm{mm})$ & $\mathrm{L}(\mathrm{mm})$ & Material & ST $(\mathrm{mm})$ & Drug & ALN (\%) \\
\hline Medtronic & Endeavor Resolute & 2.25 & 12 & $\mathrm{CoCr}$ & 0.091 & Zotarolimus & 45 \\
\hline Medtronic & Endeavor Resolute & 2.25 & 8 & $\mathrm{CoCr}$ & 0.091 & Zotarolimus & 44 \\
\hline Medtronic & Micro-Driver & 2.25 & 24 & Cobalt alloy & 0.091 & & 41 \\
\hline Abbott Vascular & Multi-Link Mini Vision & 2.50 & 28 & Cobalt alloy & 0.081 & & 41 \\
\hline Abbott Vascular & Multi-Link Mini Vision & 2.50 & 18 & Cobalt alloy & 0.081 & & 40 \\
\hline Biotronik & PRO-Kinetic & 2.50 & 22 & $\mathrm{CoCr}+\mathrm{SiC}$ coating & 0.060 & & 53 \\
\hline Cordis & Cypher Select Plus & 2.50 & 18 & $316 L$ & 0.140 & Sirolimus & 43 \\
\hline Medtronic & Endeavor Resolute & 2.50 & 12 & $\mathrm{CoCr}$ & 0.091 & Zotarolimus & 36 \\
\hline Medtronic & Endeavor Resolute & 2.50 & 24 & $\mathrm{CoCr}$ & 0.091 & Zotarolimus & 32 \\
\hline Medtronic & Micro-Driver & 2.50 & 24 & Cobalt alloy & 0.091 & & 36 \\
\hline Terumo & Tsunami Gold & 2.50 & 20 & $316 \mathrm{~L}$ & 0.080 & & 43 \\
\hline Abbott Vascular & Multi-Link Vision & 2.75 & 8 & $\mathrm{CoCr}$ & 0.081 & & 36 \\
\hline Medtronic & Endeavor Resolute & 2.75 & 8 & $\mathrm{CoCr}$ & 0.091 & Zotarolimus & 33 \\
\hline Medtronic & Endeavor Resolute & 2.75 & 30 & $\mathrm{CoCr}$ & 0.091 & Zotarolimus & 35 \\
\hline Medtronic & Endeavor Sprint & 2.75 & 24 & $\mathrm{CoCr}$ & 0.091 & Zotarolimus & 33 \\
\hline Medtronic & Micro-Driver & 2.75 & 18 & Cobalt alloy & 0.091 & & 33 \\
\hline Medtronic & Micro-Driver & 2.75 & 24 & Cobalt alloy & 0.091 & & 36 \\
\hline B. Braun & Coroflex Blue & 3.00 & 19 & $\mathrm{CoCr}$ & 0.065 & & 41 \\
\hline B. Braun & Coroflex Please & 3.00 & 19 & $316 \mathrm{~L}$ & 0.120 & Paclitaxel & 40 \\
\hline Biotronik & PRO-Kinetic & 3.00 & 30 & $\mathrm{CoCr}+\mathrm{SiC}$ coating & 0.060 & & 50 \\
\hline Boston Scientific & Liberté & 3.00 & 20 & $316 \mathrm{~L}$ & 0.097 & & 38 \\
\hline Boston Scientific & Liberté & 3.00 & 16 & $316 \mathrm{~L}$ & 0.097 & & 44 \\
\hline Boston Scientific & Taxus Liberté & 3.00 & 16 & $316 \mathrm{~L}$ & 0.097 & Paclitaxel & 39 \\
\hline Boston Scientific & Taxus Liberté & 3.00 & 20 & $316 \mathrm{~L}$ & 0.097 & Paclitaxel & 40 \\
\hline Cordis & Presillion & 3.00 & 17 & $\mathrm{CoCr}$ & 0.073 & & 38 \\
\hline Cordis & Presillion & 3.00 & 12 & $\mathrm{CoCr}$ & 0.073 & & 39 \\
\hline Medtronic & Driver & 3.00 & 12 & Cobalt alloy & 0.091 & & 40 \\
\hline Medtronic & Endeavor Resolute & 3.00 & 12 & $\mathrm{CoCr}$ & 0.091 & Zotarolimus & 44 \\
\hline MSM & Experimental* & 3.00 & 16 & $316 \mathrm{~L}+$ Tantal coating & 0.080 & & 38 \\
\hline Terumo & Tsunami Gold & 3.00 & 18 & $316 L$ & 0.080 & & 42 \\
\hline Terumo & Tsunami Gold & 3.00 & 18 & $316 \mathrm{~L}$ & 0.080 & & 39 \\
\hline Translumina & Yukon* & 3.00 & 18 & $316 L$ & 0.115 & & 41 \\
\hline Translumina & Yukon Choice* & 3.00 & 18 & $316 \mathrm{~L}$ & 0.097 & & 40 \\
\hline Translumina & Yukon Choice CC* & 3.00 & 18 & $316 L$ & 0.100 & & 41 \\
\hline Abbott Vascular & Multi-Link Vision & 3.50 & 28 & $\mathrm{CoCr}$ & 0.081 & & 25 \\
\hline Abbott Vascular & Multi-Link Vision & 3.50 & 12 & $\mathrm{CoCr}$ & 0.081 & & 33 \\
\hline Biotronik & PRO-Kinetic & 3.50 & 20 & $\mathrm{CoCr}+\mathrm{SiC}$ coating & 0.080 & & 27 \\
\hline Cordis & Cypher Select Plus & 3.50 & 13 & $316 \mathrm{~L}$ & 0.140 & Sirolimus & 32 \\
\hline Cordis & Cypher Select Plus & 3.50 & 18 & $316 L$ & 0.140 & Sirolimus & 30 \\
\hline Medtronic & Driver & 3.50 & 9 & Cobalt alloy & 0.091 & & 30 \\
\hline Medtronic & Endeavor & 3.50 & 9 & $\mathrm{CoCr}$ & 0.091 & & 30 \\
\hline Medtronic & Endeavor Resolute & 3.50 & 24 & $\mathrm{CoCr}$ & 0.091 & Zotarolimus & 22 \\
\hline Medtronic & Endeavor Resolute & 3.50 & 9 & $\mathrm{CoCr}$ & 0.091 & Zotarolimus & 25 \\
\hline Medtronic & Endeavor Sprint & 3.50 & 24 & $\mathrm{CoCr}$ & 0.091 & Zotarolimus & 21 \\
\hline
\end{tabular}


Table 1 List of coronary artery stents (Continued)

\begin{tabular}{|c|c|c|c|c|c|c|c|}
\hline Medtronic & Endeavor Sprint & 3.50 & 18 & $\mathrm{CoCr}$ & 0.091 & Zotarolimus & 25 \\
\hline Terumo & Tsunami Gold & 3.50 & 10 & $316 L$ & 0.080 & & 33 \\
\hline Terumo & Tsunami Gold & 3.50 & 18 & $316 L$ & 0.080 & & 30 \\
\hline Biotronik & PRO-Kinetic & 4.00 & 20 & $\mathrm{CoCr}+\mathrm{SiC}$ coating & 0.080 & & 29 \\
\hline Medtronic & Driver & 4.00 & 9 & Cobalt alloy & 0.091 & & 29 \\
\hline Medtronic & Driver & 4.00 & 24 & Cobalt alloy & 0.091 & & 33 \\
\hline Medtronic & Driver & 4.00 & 12 & Cobalt alloy & 0.091 & & 31 \\
\hline
\end{tabular}

*stent without a delivery system which was expanded using a balloon catheter.

List of different manufacturers, stent types, dimensions, materials and drugs of the 51 applied coronary artery stents. In addition, the ALN values of every stent using the XCD kernel are provided.

$\varnothing$ : nominal stent diameter.

L: stent length.

CoCr: cobalt chrome.

SiC: silicon carbide.

ST: strut thickness.

ALN: artificial lumen narrowing (given as percentage of the nominal lumen diameter).

\section{Attenuation and image noise}

Attenuation was defined as the difference between stent lumen attenuation and tube lumen attenuation:

$$
\text { Attenuation }=\text { SLA-TLA }
$$

We defined image noise as the mean value of the standard deviations of three axial ROIs which were positioned outside the tube lumen in the oily fluid.

\section{Statistical methods}

Data were analyzed with a statistical software (MedCalc Statistical Software, V 11.4.1.0, MedCalc bvba, Belgium). Normal distribution was assessed applying the KolmogorovSmirnov test. We used an analysis of variance for the comparison of the artificial lumen narrowing values of different groups.

As some of the attenuation values were not distributed normally, we applied the non-parametric Friedman test for the comparison of the different kernels. For the comparison of different stent size groups regarding the attenuation, the Mann-Whitney test was used as the stents of different groups were not paired.

Statistical significance was defined at $\mathrm{p}<0.05$ after application of the Bonferroni adjustment for multiple comparisons.

\section{Results}

\section{Artificial lumen narrowing}

The mean ALN values for the different reconstruction kernels and groups are listed in Table 2. In addition, a list of the ALN values of all stents as measured applying the XCD kernel is provided in Table 1.

Collectively, the XCD kernel showed markedly lower ALN values than all other kernels $(\mathrm{p}<0.001)$. Also the $\mathrm{XCB}$ kernel was significantly different to all other reconstruction algorithms $(\mathrm{p}<0.001)$. Between the $C C$ and CD kernel no significant differences were observed (p > 0.05).

ALN values for group A versus group B stents were not significantly different $(p>0.05)$ for all kernels in contrast to group $\mathrm{C}$ stents which showed a significantly lower value $(\mathrm{p}<0.001)$.

Figure 1 shows an example of a stent reconstructed with various reconstruction kernels. Figure 2 demonstrates exemplarily the effect of the stent diameter by showing three different sizes of two stent types.

\section{Halo effect}

Especially the XCD kernel yielded comparatively low ALN values for the small group A stents (see Figure 3). However, the apparently well-definable stent boundaries were visually hampered by what we labeled as 'halo effect': an artificially increased in-stent lumen image signal in the vicinity of the strut-lumen interface that decreases gradually towards the stent lumen center. An example of the halo effect is given in Figure 4.

\section{Attenuation}

The median attenuation values with the respective interquartile range (IQR) of all kernels and groups are summarized in Table 3.

Collectively, the CC kernel (18 IQR $183 \mathrm{HU}$ ) showed significantly the lowest attenuation values followed by

Table 2 Artificial lumen narrowing

\begin{tabular}{lllll}
\hline Group & XCD & CC & CD & XCB \\
\hline All & $36(21 / 53) \pm 7$ & $45(26 / 79) \pm 11$ & $45(29 / 71) \pm 10$ & $42(26 / 73) \pm 10$ \\
A & $39(32 / 53) \pm 6$ & $52(37 / 79) \pm 12$ & $51(39 / 71) \pm 9$ & $48(37 / 73) \pm 10$ \\
B & $41(38 / 58) \pm 3$ & $50(44 / 54) \pm 3$ & $49(36 / 57) \pm 6$ & $46(40 / 53) \pm 4$ \\
C & $29(21 / 33) \pm 4$ & $35(26 / 44) \pm 5$ & $34(29 / 46) \pm 5$ & $32(26 / 42) \pm 5$ \\
\hline
\end{tabular}

Artificial lumen narrowing values are given as mean (minimum/maximum) \pm standard deviation in percent of the nominal lumen diameter. 


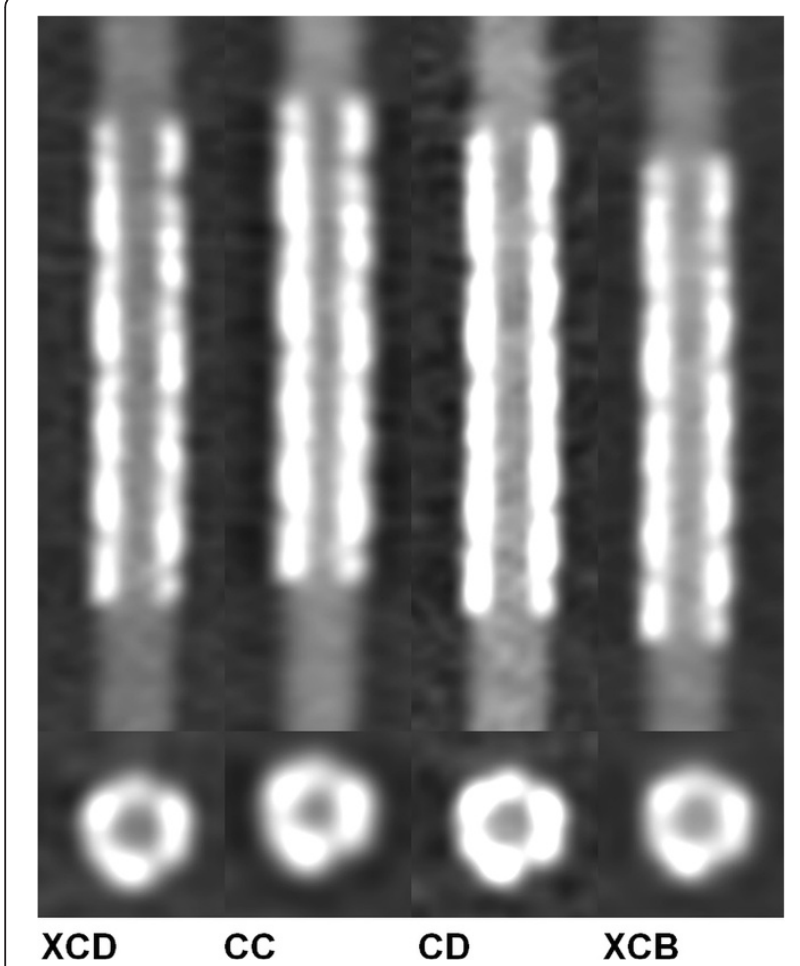

Figure 1 Differences of reconstruction kernels. Reconstructions of the same stent applying the four different kernels. (B. Braun Coroflex Please, nominal diameter $3.0 \mathrm{~mm}$, length $19 \mathrm{~mm}$ ).

the CD kernel (40 IQR $96 \mathrm{HU})$. While the difference between $\mathrm{CD}$ and XCD (43 IQR $34 \mathrm{HU}$ ) yielded no significance, the $\mathrm{XCB}$ method showed a significantly higher value (128 IQR $122 \mathrm{HU}$ ) than all other kernels.

Consequently, we performed an analysis of the different stent size groups.

For group A stents, the discrepancy between all kernels was significant with the XCD kernel providing values closer to the optimum of zero than the other ones.

In group $\mathrm{B}, \mathrm{XCD}$ showed no significant differences to $\mathrm{CD}$ whereas all other differences including those to $\mathrm{XCB}$ were significant. $\mathrm{CC}$ showed the best attenuation values followed by XCD.

In group $\mathrm{C}$, only the difference between $\mathrm{XCD}$ and $\mathrm{XCB}$ was not significant with $\mathrm{CD}$ providing the attenuation values closest to the optimum of zero.

Whereas the values for XCD remained quite constant and only declined about $13 \mathrm{HU}$ from group $\mathrm{A}$ to $\mathrm{C}$, the other groups showed notably higher drops up to 240 HU for the CC kernel (see Figure 5). The CC and the $\mathrm{CD}$ kernel even showed negative values.

\section{Noise}

As noise was measured independently from the stent diameter a sub-categorization into different groups was not reasonable. The soft XCB kernel showed significantly
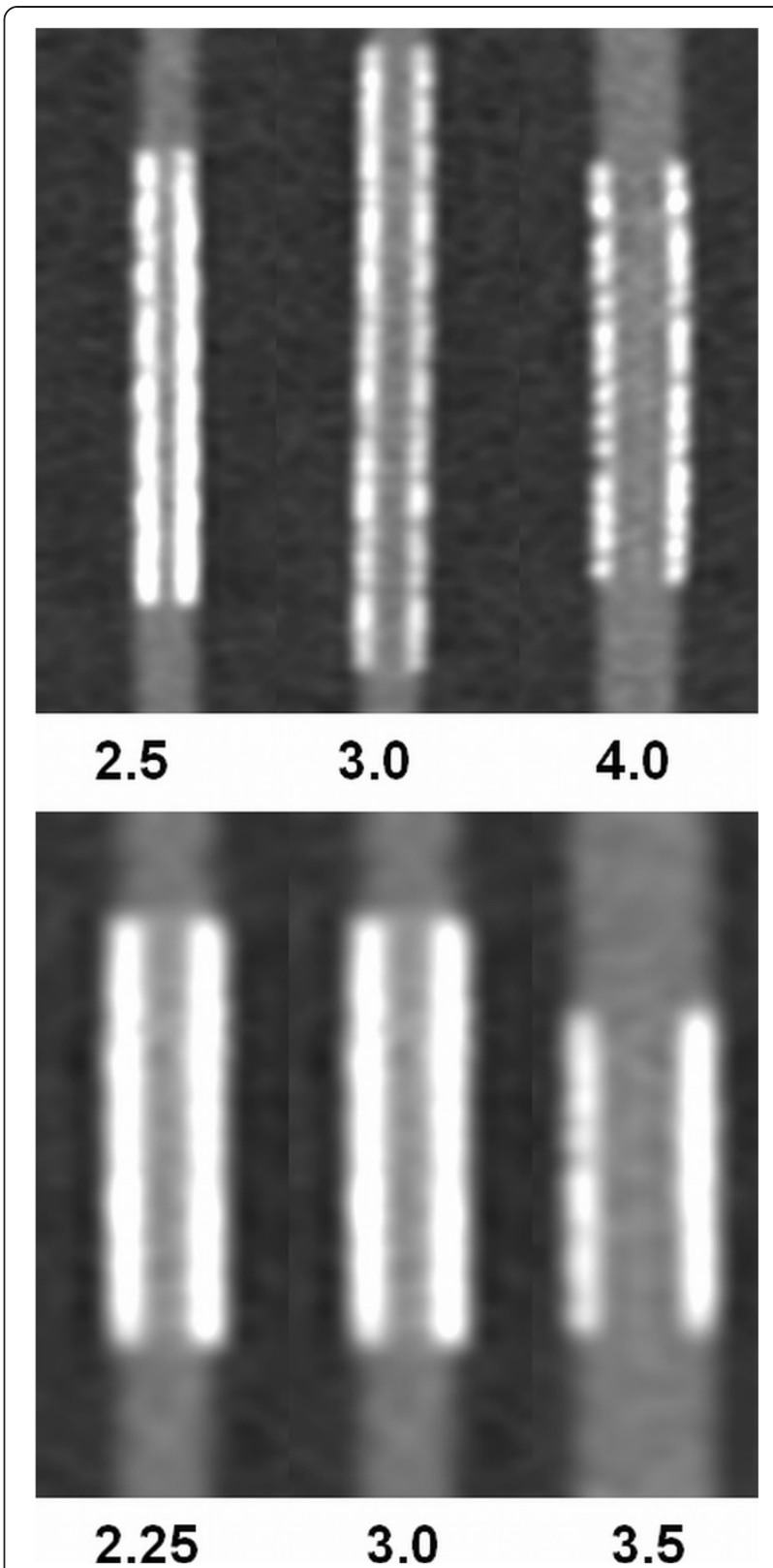

3.0

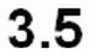

Figure 2 Effect of different stent diameter. Reconstructions of three different sizes of the same stent type (top: Biotronik PRO-Kinetic, bottom: Medtronic Endeavor Resolute). Diameter is given in $\mathrm{mm}$. Zoom factors differ between the stent types.

lower values than all other kernels $(15.8 \pm 1.9 \mathrm{HU})$. Of the remaining kernels, the $\mathrm{CD}$ kernel had the highest values $(34.9 \pm 4.6 \mathrm{HU})$, whereas values for the XCD $(21.1 \pm 2.9$ $\mathrm{HU})$ and CC (19.9 $\pm 2.2 \mathrm{HU})$ kernels were comparable. Yet the differences between all kernels were statistically significant (all $\mathrm{p}<0.001$ ).

\section{Discussion}

In a state of the art 256-MSCT scanner four different reconstruction kernels were employed to scan 51 


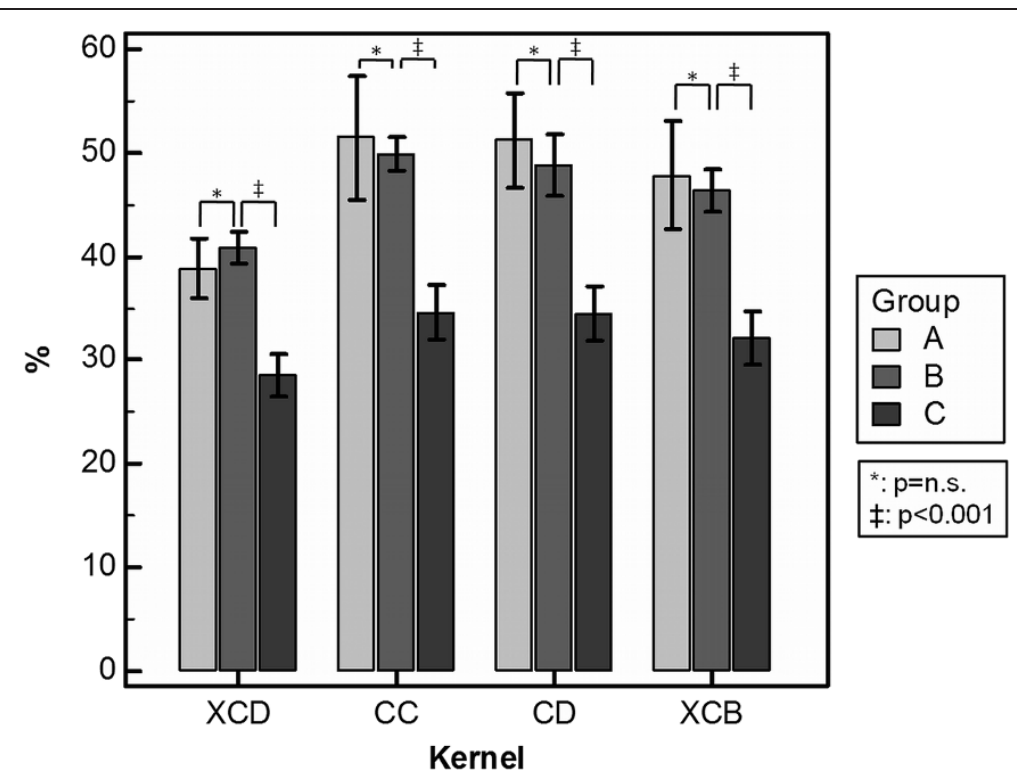

Figure 3 Artificial lumen narrowing. Comparison of the ALN values of different stent groups applying the four reconstruction kernels.

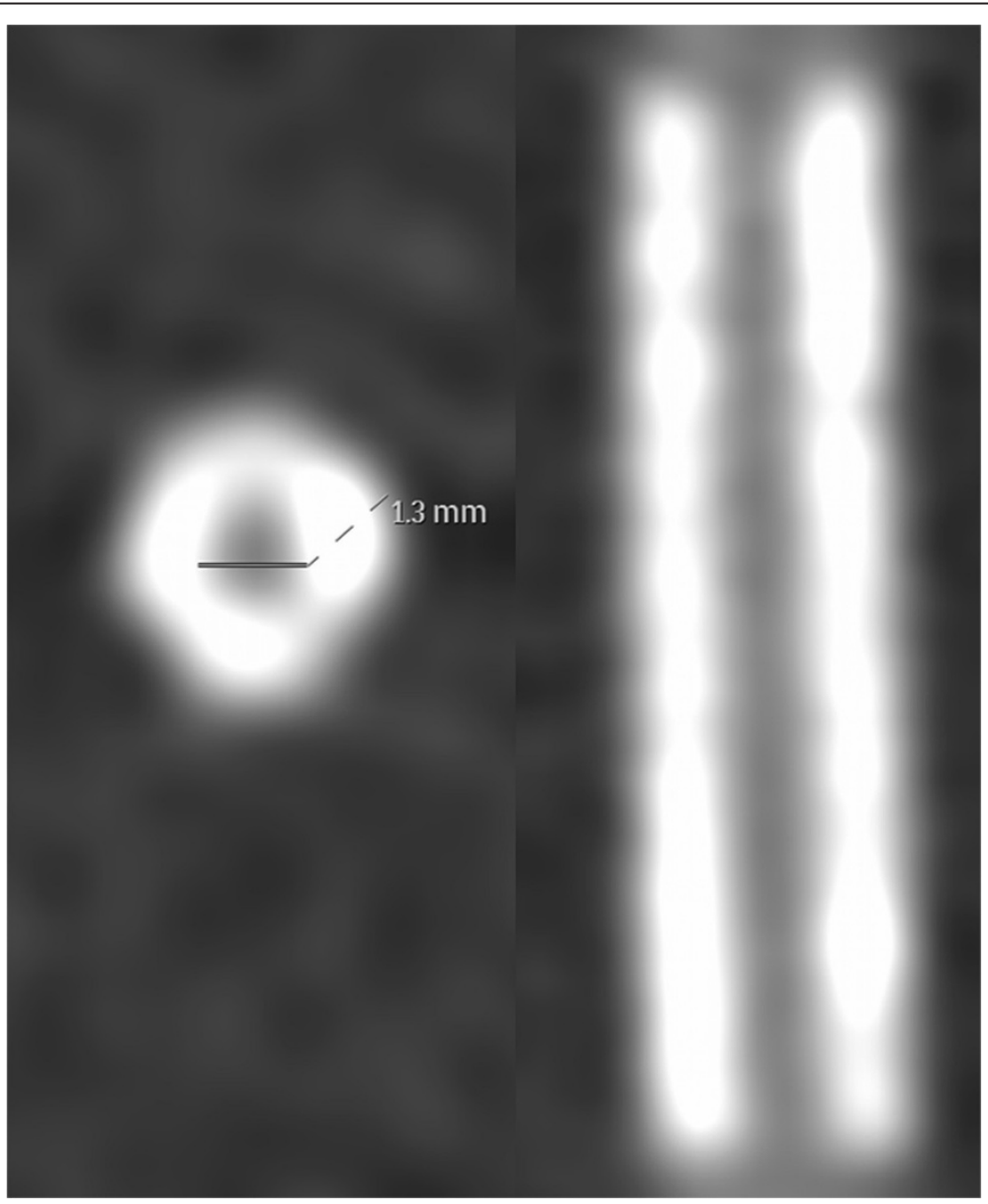

Figure 4 Example of the halo effect. The ALN for this stent was manually measured as $45 \%$. However, the lumen visualization was considerably impaired by the halo effect (Medtronic Endeavor Resolute, nominal diameter $2.25 \mathrm{~mm}$, length $12 \mathrm{~mm}$ ). 
Table 3 Attenuation

\begin{tabular}{lllll}
\hline Group & XCD & CC & CD & XCB \\
\hline All & $43(22 / 174)_{\mathrm{IQR}} 34$ & $18(-177 / 392)_{\mathrm{IQR}} 183$ & $40(-78 / 254)_{\mathrm{IQR}} 96$ & $128(6 / 396)_{\mathrm{IQR}} 122$ \\
A & $55(22 / 174)_{\mathrm{IQR}} 55$ & $127(14 / 392)_{\mathrm{IQR}} 186$ & $78(14 / 254)_{\mathrm{IQR}} 34$ & $189(116 / 396)_{\mathrm{IQR}} 158$ \\
B & $41(27 / 125)_{\mathrm{IQR}} 48$ & $36(-2 / 151)_{\mathrm{IQR}} 65$ & $48(9 / 153)_{\mathrm{IQR}} 56$ & $131(87 / 265)_{\mathrm{IQR}} 59$ \\
C & $42(22 / 116)_{\mathrm{IQR}} 17$ & $-113(-177 /-18)_{\mathrm{IQR}_{\mathrm{R}}} 35$ & $-29(-78 / 33)_{\mathrm{IQR}} 29$ & $49(6 / 163)_{\mathrm{IQR}} 33$ \\
\hline
\end{tabular}

Attenuation values are given as median (minimum/maximum) interquartile range in $\mathrm{HU}$.

coronary artery stents. Recently published clinical studies could show that the quantification of atherosclerotic coronary lumen narrowing as well as the assessment of plaque is feasible with this scanner generation [17]. However, to date there is only scarce data regarding the potential of the 256-MSCT for the visualization of the coronary stent lumen $[1,18,19]$. In contrast to our first technical oriented studies, we applied a clinically applicable approach.

The applied XCB kernel is the standard algorithm for the assessment of coronary arteries in Philips CT scanners. For the reconstruction of coronary stents the $\mathrm{CD}$ and XCD kernel are provided by the manufacturer. Although both kernels have similar reconstruction characteristics, the XCD features a reduced image noise. In previous studies concerning stent visualization the B46f kernel has been recommended for the 64-MSCT and DSCT from Siemens $[15,16]$ and the Q04 kernel for Toshiba's 64-MSCT [20].

Overall and in all stent groups, the Philips XCD kernel showed lower ALN values than the other three kernels. The edge-enhancing characteristic of the XCD's reconstruction method provided sufficiently visible strut margins, so that the electronic caliper could be positioned precisely in most measurements. Consequently, the ALN standard deviations of the XCD kernel were smaller compared to the other three kernels. A similar superiority of the XCD kernel became evident with respect to the attenuation. The measured attenuation values applying the XCD kernel were consistent across the stent size groups whereas the attenuation for the CC and CD kernels declined from highly positive values in small stents to even negative measurements in larger stents. The phenomenon of radiodensity values being lower in the stent than in the tube lumen has been described before $[1,15,21]$. One may speculate that the sharpening algorithms of these kernels could be responsible for this effect. However, the diagnostic value and interpretation of negative attenuation values within the stent lumen is unclear and needs further investigation. Although the $\mathrm{CD}$ kernel showed attenuation values closer to the optimum of zero in group $\mathrm{C}$ stents, it was accompanied by considerable higher noise and ALN.

As the XCB kernel is softer than the others, it provided significantly lower noise values. Yet, the noise difference between the XCD and the XCB kernels was only about $5 \mathrm{HU}$ and therefore negligible.

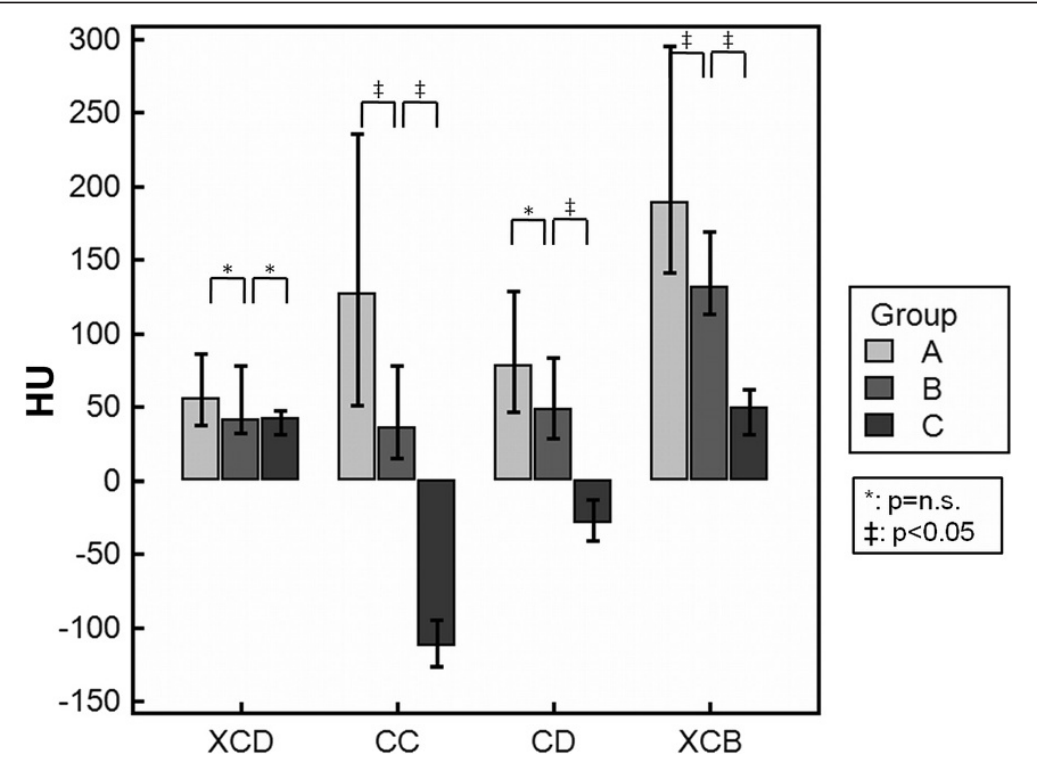

Figure 5 Attenuation. Attenuation values as provided by the different reconstruction kernels. Note that with increasing stent size the values of the CC and CD kernels become negative. 
In summary, the XCD kernel provided the most reliable ALN and attenuation values at the cost of a little higher noise. Our first conclusion therefore is that we recommend the application of the XCD kernel in addition to the routine $\mathrm{XCB}$ kernel when examining a patient with a coronary artery stent.

We investigated various coronary artery stents of different sizes, designs, materials and manufacturers. As most of the coronary stents used in clinical routine are either made of $316 \mathrm{~L}$ stainless steel or cobalt chrome, the stent size and architecture is, in a majority of cases, a more important factor for the quality of stent lumen visualization than the material.

At first glance, the ALN values for group A and B stents seemed to be astonishingly low with most values being $<50 \%$ using the XCD kernel. However, the visibility of their lumina was impaired by a phenomenon we introduced as 'halo effect' (Figure 4). This phenomenon resembles the so-called blooming artifact but unlike this it is rather characterized by altered attenuation values inside the stent than by an exaggerated thickness of the stent struts as described for blooming [22,23]. The halo effect seemed to be more pronounced in smaller stents, especially when reconstructed with harder kernels. Although the struts were clearly visible the assessability of the adjacent lumen was impaired. As the voxel signals at the interface between tube lumen and stent struts seemed to be artificially smeared, atherosclerotic processes like ISR could potentially be missed or misinterpreted. Clinically, the halo effect could therefore often lead to false diagnoses. Therefore, we advise against the employment of coronary CT for the exclusion of ISR in stents $<3 \mathrm{~mm}$ due to the halo effect.

In agreement with our findings, in a lately published meta-analysis on 64-MSCT, Carrabba et al. stated that the rate of uninterpretable stent lumina tended to be more pronounced for stent diameters $<3 \mathrm{~mm}$ [24]. Likewise, in a recent review about the diagnostic accuracy of 64-MSCT for the detection of ISR, Sun et al. concluded that stent lumen evaluation should be restricted to larger stents $(>3 \mathrm{~mm})$ [11]. Since these measurements were generated using 64-MSCT, state of the art scanner generations such as DSCT or 256-MSCT have encouraged the expectation that ISR in smaller stents could accurately be detected or excluded. And indeed, Pugliese et al., in a clinical DSCT study in which stent lumen assessment of different sizes was investigated, showed that the negative predictive value was $100 \%$ for stents $\geq 3 \mathrm{~mm}$ and $90 \%$ for stents $\leq 2.75 \mathrm{~mm}$ [25]. Although stent diameters $\leq 2.75 \mathrm{~mm}$ lead to frequently false positive results, the authors drew the conclusion that DSCT coronary angiography could reliably rule out ISR irrespective of the stent size due to its high negative predictive value. In another clinical DSCT study all stent lumen with a diameter $>3 \mathrm{~mm}$ were assessable, whereas only $81 \%$ of stents with a caliber of $3 \mathrm{~mm}$ could be evaluated [26].

To date, there is only one clinical pilot study assessing the diagnostic capability of the 256-MSCT for ISR detection in 28 patients [18]. In this study, Oda et al. attained a sensitivity and specificity of $100 \%$ and $55 \%$ by combining the $\mathrm{CD}$ kernel with an iterative reconstruction algorithm leading to positive and negative predictive values of $40 \%$ and $100 \%$. Yet, the authors state the limitation that the diagnostic performance of the 256-MSCT in different stent sizes and types was not evaluated. In our in-vitro study, all stents with a diameter of $3 \mathrm{~mm}$, that were reconstructed with the XCD kernel, showed an ALN that did not exceed 50\%, which is sufficient to exclude a significant luminal stenosis $>50 \%$. Compared to group A stents $(<3 \mathrm{~mm})$, the extent of the halo effect was clearly less pronounced in group B $(3 \mathrm{~mm})$. Therefore, clinical in-vivo stent assessment might be possible for group B stents using the XCD kernel. However, future in-vitro and in-vivo studies are required to examine the possibility of stent lumen visualization for stents with a diameter of $3 \mathrm{~mm}$ in 256-MSCT.

Group C stents $(>3 \mathrm{~mm}$ ) revealed significantly lower ALN values and showed a negligible extent of the halo effect. These findings are in line with previously published literature and leads us to recommend 256MSCT for the stent lumen assessment of coronary stents with a lumen diameter larger than $3.0 \mathrm{~mm}$ $[1,11,21,26,27]$.

\section{Study limitations}

Although the phantom was designed to mimic in-vivo conditions and has been used in a previous study, several limitations have to be considered.

First, all stents were scanned in an orientation parallel to the scanner's z-axis. Previous studies have indicated that the quality of stent visualization may depend on the stent and scanner angulations [28,29].

Second, since a static phantom with an artificial continuous heart rate of $60 \mathrm{bpm}$ was used, cardiac and respiratory movements could not be simulated. Therefore, high temporal resolution techniques of the 256MSCT like fast rotation time and wide $\mathrm{z}$-axis coverage were not regarded.

Third, the scan parameters in this study were chosen similar to the protocols for clinical routine. We did not evaluate the potential of advanced dose-saving algorithms for stent visualization. This may be an objective of further studies.

Fourth, the window settings were chosen in accordance with previous studies [1,30]. Yet, these settings indeed influence the visual measurement of the stent lumen diameter, as the visible strut thickness may vary at different settings. 
Last, since the experimental set-up differed from other phantom studies concerning coronary stent visualization, the results are not fully comparable.

\section{Conclusions}

With the 256-MSCT, the assessment of stent lumina larger than $3 \mathrm{~mm}$ is clinically possible if stent-dedicated kernels are applied. The XCD reconstruction kernel proved to be superior for lumen visualization independently from the stent caliber. The stent lumen visualization of stents with a diameter of $3 \mathrm{~mm}$ seems to be feasible but has to be validated in further studies.

Additional investigations are needed to assess the visualization of stents with a lumen diameter smaller than $3 \mathrm{~mm}$. Further improvements in stent designs and materials as well as in CT spatial resolution and reconstruction methods could help accomplish the assessment of coronary stents using CT as part of the clinical routine.

\section{Availability of supporting data}

The data sets supporting the results of this article are included within the article and its additional files.

\begin{abstract}
Abbreviations
ALN: Artificial lumen narrowing; BMS: Bare metal stents; CA: Coronary angiography; CAD: Coronary artery disease; CoCr: Cobalt chrome; CT: Computed tomography; DES: Drug eluting stents; DSCT: Dual source computed tomography; IQR: Interquartile range; ISR: In-stent restenosis; MSCT: Multislice computed tomography; PCI: Percutaneous coronary intervention; ROI: Region of interest; SiC: Silicon carbide; SLA: Stent lumen attenuation; TLA: Tube lumen attenuation.
\end{abstract}

\section{Competing interests}

The authors declare that they have no competing interests.

\section{Authors' contributions}

FA and HS designed the trial, analyzed and interpreted the data and wrote the manuscript. FA performed the data acquisitions. DM contributed to the design of the study and drafted the manuscript. GM, WM and HUK have been involved in drafting and revising the manuscript. HK revised the manuscript critically and gave the final approval. HS also gave the final approval for this manuscript to be published. All authors read and approved the final manuscript.

\section{Author details}

${ }^{1}$ Department of Cardiology, University of Heidelberg, Im Neuenheimer Feld 410, Heidelberg 69120, Germany. ${ }^{2}$ Philips GmbH Healthcare Division, Luebeckertordamm 5, Hamburg 20099, Germany. ${ }^{3}$ Department of Diagnostic and Interventional Radiology, University of Heidelberg, Im Neuenheimer Feld 410, Heidelberg 69120, Germany.

Received: 1 August 2013 Accepted: 30 December 2013

Published: 14 January 2014

\section{References}

1. Steen H, André F, Korosoglou G, Mueller D, Hosch W, Kauczor H-U, Giannitsis $\mathrm{E}$, Katus HA: In vitro evaluation of 56 coronary artery stents by 256-slice multi-detector coronary CT. Eur J Radiol 2011, 80:143-150.

2. Go AS, Mozaffarian D, Roger VL, Benjamin EJ, Berry JD, Borden WB, Bravata DM, Dai S, Ford ES, Fox CS, et al: Heart disease and stroke statistics-2013 update: a report from the American Heart Association. Circulation 2013, 127:e6-e245.
3. Lagerqvist B, James SK, Stenestrand U, Lindback J, Nilsson T, Wallentin L: Long-term outcomes with drug-eluting stents versus bare-metal stents in Sweden. N Engl J Med 2007, 356:1009-1019.

4. Dangas GD, Claessen BE, Caixeta A, Sanidas EA, Mintz GS, Mehran R: In-stent restenosis in the drug-eluting stent era. J Am Coll Cardiol 2010, 56:1897-1907.

5. Lemesle G, Maluenda G, Collins SD, Waksman R: Drug-eluting stents: issues of late stent thrombosis. Cardiol Clin 2010, 28:97-105.

6. Mowatt G, Cook JA, Hillis GS, Walker S, Fraser C, Jia X, Waugh N: 64-Slice computed tomography angiography in the diagnosis and assessment of coronary artery disease: systematic review and meta-analysis. Heart 2008, 94:1386-1393.

7. Sun Z, Lin C, Davidson R, Dong C, Liao Y: Diagnostic value of 64-slice CT angiography in coronary artery disease: a systematic review. Eur J Radiol 2008, 67:78-84.

8. Schmermund A, Haude M, Baumgart D, Gorge G, Gronemeyer D, Seibel R, Sehnert C, Erbel R: Non-invasive assessment of coronary Palmaz-Schatz stents by contrast enhanced electron beam computed tomography. Eur Heart J 1996, 17:1546-1553.

9. Krüger S: Multislice spiral computed tomography for the detection of coronary stent restenosis and patency. Int J Cardiol 2003, 89:167-172.

10. Sun Z, Davidson R, Lin CH: Multi-detector row CT angiography in the assessment of coronary in-stent restenosis: a systematic review. Eur J Radiol 2009, 69:489-495.

11. Sun Z, Almutairi AM: Diagnostic accuracy of 64 multislice CT angiography in the assessment of coronary in-stent restenosis: a meta-analysis. Eur J Radiol 2010, 73:266-273.

12. Kumbhani DJ, Ingelmo CP, Schoenhagen P, Curtin RJ, Flamm SD, Desai MY: Meta-analysis of diagnostic efficacy of 64-slice computed tomography in the evaluation of coronary in-stent restenosis. Am J Cardiol 2009, 103:1675-1681.

13. Hamon M, Champ-Rigot L, Morello R, Riddell JW: Diagnostic accuracy of in-stent coronary restenosis detection with multislice spiral computed tomography: a meta-analysis. Eur Radiol 2008, 18:217-225.

14. Vanhoenacker PK, Decramer I, Bladt O, Sarno G, Van Hul E, Wijns W, Dwamena BA: Multidetector computed tomography angiography for assessment of in-stent restenosis: meta-analysis of diagnostic performance. BMC Med Imaging 2008, 8:14.

15. Maintz D, Burg MC, Seifarth H, Bunck AC, Ozgun M, Fischbach R, Jurgens $\mathrm{KU}$, Heindel W: Update on multidetector coronary $C T$ angiography of coronary stents: in vitro evaluation of 29 different stent types with dual-source CT. Eur Radiol 2009, 19:42-49.

16. Maintz D, Seifarth $H$, Raupach R, Flohr T, Rink M, Sommer T, Ozgun M, Heindel W, Fischbach R: 64-slice multidetector coronary CT angiography: in vitro evaluation of 68 different stents. Eur Radiol 2006, 16:818-826.

17. Korosoglou G, Mueller D, Lehrke S, Steen H, Hosch W, Heye T, Kauczor HU, Giannitsis E, Katus HA: Quantitative assessment of stenosis severity and atherosclerotic plaque composition using 256-slice computed tomography. Eur Radio/ 2010, 20:1841-1850.

18. Oda S, Utsunomiya D, Funama Y, Takaoka H, Katahira K, Honda K, Noda K, Oshima S, Yamashita Y: Improved coronary in-stent visualization using a combined high-resolution kernel and a hybrid iterative reconstruction technique at 256-slice cardiac CT-Pilot study. Eur J Radio/ 2013, 82:288-295.

19. Andre F, Korosoglou G, Hosch W, Giannitsis E, Kauczor HU, Katus HA Steen $\mathrm{H}$ : Performance of dual source versus 256-slice multi-slice CT in the evaluation of 16 coronary artery stents. Eur J Radiol 2013, 82:601-607.

20. Schuijf JD, Pundziute G, Jukema JW, Lamb HJ, Tuinenburg JC, van der Hoeven BL, de Roos A, Reiber JH, van der Wall EE, Schalij MJ, Bax JJ: Evaluation of patients with previous coronary stent implantation with 64-section CT. Radiology 2007, 245:416-423.

21. Wolf F, Cademartiri F, Loewe C, Stadler A, Weber M, Lammer J, Feuchtner GM: Evaluation of coronary stents with 64-MDCT: in vitro comparison of scanners from four vendors. Am J Roentgenol 2009, 193:787-794.

22. Maintz D, Juergens KU, Wichter T, Grude M, Heindel W, Fischbach R: Imaging of coronary artery stents using multislice computed tomography: in vitro evaluation. Eur Radio/ 2003, 13:830-835.

23. Nieman $K$, Cademartiri F, Raaijmakers R, Pattynama P, de Feyter P: Noninvasive angiographic evaluation of coronary stents with multi-slice spiral computed tomography. Herz 2003, 28:136-142. 
24. Carrabba N, Schuijf JD, de Graaf FR, Parodi G, Maffei E, Valenti R, Palumbo A, Weustink AC, Mollet NR, Accetta G, et al: Diagnostic accuracy of 64-slice computed tomography coronary angiography for the detection of in-stent restenosis: a meta-analysis. J Nucl Cardiol 2010, 17:470-478.

25. Pugliese F, Weustink AC, Van Mieghem C, Alberghina F, Otsuka M, Meijboom WB, van Pelt N, Mollet NR, Cademartiri F, Krestin GP, et al: Dual source coronary computed tomography angiography for detecting in-stent restenosis. Heart 2008, 94:848-854.

26. Pflederer T, Marwan M, Renz A, Bachmann S, Ropers D, Kuettner A, Anders K, Bamberg F, Daniel WG, Achenbach S: Noninvasive assessment of coronary in-stent restenosis by dual-source computed tomography. Am J Cardiol 2009, 103:812-817.

27. Wykrzykowska JJ, Arbab-Zadeh A, Godoy G, Miller JM, Lin S, Vavere A, Paul N, Niinuma H, Hoe J, Brinker J, et al: Assessment of in-stent restenosis using 64-MDCT: analysis of the CORE-64 Multicenter International Trial. AIR Am J Roentgenol 2010, 194:85-92.

28. Seifarth H, Ozgun M, Raupach R, Flohr T, Heindel W, Fischbach R, Maintz D: 64- Versus 16-slice $C T$ angiography for coronary artery stent assessment: in vitro experience. Invest Radiol 2006, 41:22-27.

29. Donati OF, Burg MC, Desbiolles L, Karlo C, Stolzmann P, Bunck A, Baumueller S, Marincek B, Seifarth H, Alkadhi H, et al: High-pitch 128-slice Dual-source $\mathrm{CT}$ for the assessment of coronary stents in a phantom model. Acad Radiol 2010, 17:1366-1374.

30. Haraldsdottir S, Gudnason T, Sigurdsson AF, Gudjonsdottir J, Lehman SJ, Eyjolfsson K, Scheving SS, Gibson CM, Hoffmann U, Jonsdottir B, Andersen K: Diagnostic accuracy of 64-slice multidetector $\mathrm{CT}$ for detection of in-stent restenosis in an unselected, consecutive patient population. Eur J Radiol 2009, 76:188-194.

doi:10.1186/1756-0500-7-38

Cite this article as: André et al: In-vitro assessment of coronary artery stents in 256-multislice computed tomography angiography. BMC Research Notes 2014 7:38.

\section{Submit your next manuscript to BioMed Central and take full advantage of:}

- Convenient online submission

- Thorough peer review

- No space constraints or color figure charges

- Immediate publication on acceptance

- Inclusion in PubMed, CAS, Scopus and Google Scholar

- Research which is freely available for redistribution 